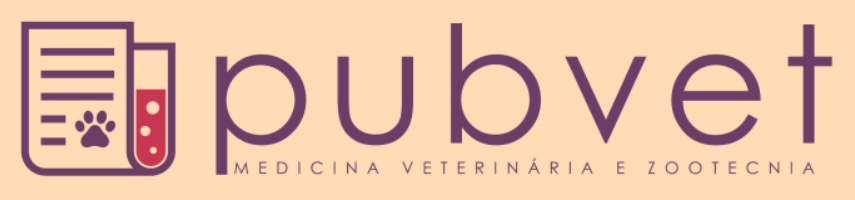

https://doi.org/10.31533/pubvet.v12n7a140.1-6

\title{
Hiperparatireoidismo secundário nutricional em felino doméstico: Relato de caso
}

\author{
Breno Costa de Macedo ${ }^{1 *}{ }^{\bullet}$, Ana Celi Santos $\operatorname{Costa}^{1}{ }^{\bullet}$, Lais Andressa Silva de Souza ${ }^{10}$, \\ Jaese Farias Chaves ${ }^{1} \bullet$, Valdelice de Lourdes Correa Pinheiro ${ }^{\circ}{ }^{\bullet}$, Katiane Schwanke ${ }^{3}{ }^{\circ}$, \\ Carolina Franchi João ${ }^{7}{ }^{\circ}$, Danilo Ferreira Rodrigues ${ }^{8} \bullet$
}

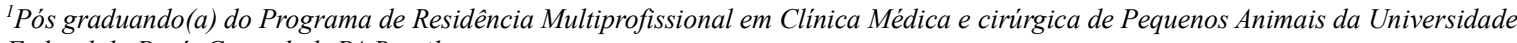
Federal do Pará. Castanhal- PA Brasil.

${ }^{2}$ Mestranda do Programa de Pesquisa e Pós-Graduação em Biologia de Agentes Infecciosos e Parasitários da Universidade Federal do Pará. Belém- PA Brasil.

${ }^{3}$ Médica Veterinária, Hospital Veterinário-Setor Pequenos Animais Universidade Federal do Pará Castanhal.-Pa Brasil.

${ }^{4}$ Professor da Universidade Federal do Pará, Instituto de Medicina Veterinária. Castanhal.-Pa Brasil. E-mail:carolfranchi@yahoo.com.br

${ }^{4}$ Professor da Universidade Federal do Pará, Instituto de Medicina Veterinária. Castanhal.-Pa Brasil. E-mail: dan rodrigues2@yahoo.com.br

*Autor para correspondência,E-mail: brenomacedo@hotmail.fr

RESUMO. O hiperparatireoidismo secundário nutricional (HSN) é uma doença metabólica caracterizada pelo desequilíbrio da relação de Ca e P, levando a osteopenia. Clinicamente se manifesta com claudicação, constipação, lordose, cifose, fraturas de ossos longos e corpos vertebrais. O presente trabalho relata um caso de HSN em um felino macho de 3 meses de idade alimentado com alimentação caseira atendido no Hospital Veterinário (HOVET) da Universidade Federal do Pará (UFPA). O mesmo apresentava claudicação que evoluiu para paraparesia com déficit neurológico nos membros pélvicos, constipação e retenção urinaria. Foi submetido a exames hematológicos, bioquímicos e radiográficos, apresentando como resultado mais significativo osteopenia severa com múltiplas fraturas patológicas. A partir dos dados clínicos e dos exames realizados obteve-se o diagnóstico de HSN. O animal foi submetido a tratamento constituído por confinamento, analgesia e correção alimentar obtendo evolução satisfatória após 30 dias e alta medica após quatro meses. A literatura apresenta-se bastante limitada quanto a dados clínicos e resultados de tratamento de felinos com HSN. Embora esta patologia venha se tornando cada vez mais incomum entre felinos ainda são necessárias contribuições que permitam tratamentos mais eficientes e prognósticos mais favoráveis.

Palavras chave: doença metabólica, carência nutricional, osteopenia, clínica de felinos

\section{Nutritional secondary hyperparathyroidism in a domestic feline: Case report}

ABSTRACT. Secondary nutritional hyperparathyroidism (HSN) is a metabolic disease characterized by imbalance of the $\mathrm{Ca}$ and $\mathrm{P}$, leading to osteopenia. The symptoms are claudication, constipation, lordosis, kyphosis, fractures of long bones and vertebral bodies. The present study reports a case of NSH in a 3-month-old male feline fed with homecare food that was attended at UFPA HOVET presenting claudication that progressed to paraparesis with neurological deficit in pelvic limbs, constipation and urinary retention. It was submitted to hematological, biochemical and radiographic examinations, presenting severe osteopenia with multiple pathological fractures, which related to the clinical data helped to obtain the diagnosis of HSN. The animal was submitted to treatment consisting of confinement, analgesia and alimentary correction and it was obtained satisfactory evolution after 30 days with high medication after 4 months. The literature is very limited as to the clinical data and results of treatment of felines with HSN, although this pathology 
is becoming increasingly uncommon among felines, contributions are still needed to allow more efficient treatments and more favorable prognoses.

Keywords: metabolic disease, nutritional deficiency, osteopenia, feline clinic

\section{Hiperparatiroidismo secundario nutricional en felino doméstico: Reporte de un caso}

RESUMEN: El hiperparatiroidismo secundario nutricional (HSN) es una enfermedad metabólica caracterizada por el desequilibrio de la relación de Ca y P, llevando a la osteopenia. Clínicamente se manifiesta con claudicación, constipación, lordosis, cifosis, fracturas de huesos largos y cuerpos vertebrales. El presente trabajo relata un caso de HSN en un felino macho de 3 meses de edad alimentado con comida casera, el paciente fue atendido en el HOVET de la UFPA. El mismo presentaba claudicación que evolucionó hacia paraparesia con déficit neurológico en los miembros pélvicos, constipación y retención urinaria. Se sometió a exámenes hematológicos, bioquímicos y radiográficos, dando como resultado más significativo la osteopenia severa con múltiples fracturas patológicas observadas en las radiografías. A partir de los datos clínicos y de los exámenes realizados se obtuvo el diagnóstico de HSN. El animal fue sometido a tratamiento constituido por confinamiento, administración de medicamentos analgésicos y corrección alimenticia obteniendo una evolución satisfactoria después de 30 días, el alta médica fue dada después de los cuatro meses. La literatura se presenta bastante limitada en cuanto a los datos clínicos y resultados de tratamiento de felinos con HSN. Aunque esta patología se está volviendo cada vez menos frecuentes en felinos, todavía son necesarias contribuciones que permitan tratamientos más eficientes y pronósticos más favorables.

Palabras claves: enfermedad metabólica, carencia nutricional, osteopenia, clínica de felinos

\section{Introdução}

O hiperparatireoidismo secundário nutricional (HSN) é uma doença óssea metabólica caracterizada pelo aumento da secreção de paratormônio compensatória ao desequilíbrio da relação de cálcio e fósforo na dieta (Jubb et al., 2007) levando a um quadro de osteopenia generalizada (Meireles et al., 2012). Esta enfermidade já foi descrita em diversas espécies domésticas (Queiroz et al., 2015) e selvagens (Meireles et al., 2012), podendo acometer felinos jovens em sua fase de crescimento ósseo mais abundante, quando há uma demanda maior pela ingestão de cálcio (Tomsa et al., 1999). Doenças nutricionais como o HSN já foram comuns na clínica de pequenos animais, no entanto, essa patologia tem se tornado raras desde a difusão de alimentos industrializados nutricionalmente equilibrados (Tomsa et al., 1999; Dimopoulou et al., 2010). Atualmente, relatos esporádicos ainda são encontrados na literatura (Rahal et al., 2002; Nagata \& Yuki, 2013; Prestes et al., 2016) mostrando que, embora seja incomum nos dias atuais, o HSN ainda constitui uma enfermidade importante na clínica de felinos.
Clinicamente caracteriza-se por claudicação, constipação, lordose, cifose, fraturas de ossos longos e corpos vertebrais (Tomsa et al., 1999; Rahal et al., 2002; Dimopoulou et al., 2010). Fraturas de corpo vertebral quando ocasionam déficit neurológico segundo a literatura possuem um prognostico ruim com recomendação de eutanásia dos animais acometidos (Tomsa et al., 1999). Outros fatores como constipação e retenção urinária também são apontados na literatura como razão para eutanásia (Dimopoulou et al., 2010) não havendo relatos sobre recuperação de animais com estas lesões.

Tendo em vista a importância do HSN para a clínica de felinos, assim como a pouca literatura disponível sobre esta enfermidade em gatos domésticos, o presente trabalho objetivou relatar o caso de um felino diagnosticado com HSN apresentando lesão na coluna vertebral com déficit neurológico e sua recuperação.

\section{Relato de caso}

Foi atendido no Hospital Veterinário da Universidade Federal do Pará um gato, sem raça definida, macho de aproximadamente três meses, 
com claudicação do membro pélvico esquerdo há dois meses e que, há duas semanas não conseguia se apoiar nos membros pélvicos. O proprietário relatou ainda que o animal não defecava há alguns dias e não urinava quando colocado na caixa de areia, aparecendo urinado esporadicamente. O mesmo não apresentava qualquer histórico de trauma e era alimentado com comida caseira constituída geralmente por carne ou peixe com arroz ou macarrão.

Mediante o exame físico, observou se que o animal estava alerta, apresentava estado nutricional regular, mucosas discretamente pálidas, cerca de $8 \%$ de desidratação, frequência cardíaca 240 BPM, frequência respiratória 100 MRM, temperatura retal de $37,7^{\circ} \mathrm{c}$ e tempo de preenchimento capilar de 2 segundos. A palpação dos membros pélvicos revelou mobilidade acentuada na região da diáfise femoral bilateralmente e $\mathrm{o}$ animal manifestava dor evidente quando palpado. Observou-se ainda ausência de propriocepção, resposta reduzida a dor superficial e profunda, além de distensão vesical e intestinal.

Foi realizado exame hematológico e bioquímico dosando cálcio total e iônico, fósforo, creatinina, proteína total e frações. O animal foi encaminhado para exames radiográficos dos membros pélvicos e coluna toracolombar.

Uma discreta leococitose $\left(20.700 \mathrm{~mm}^{3}-5.500\right.$ A $19.500 \mathrm{~mm}^{3}$ ) com neotrofilia foi observada nos exames hematológicos. $\mathrm{O}$ aumento da concentração sérica de fósforo $(7,4 \mathrm{mg} / \mathrm{dL}-1,8$ a $6,4 \mathrm{mg} / \mathrm{dL}$ ) foi a única alteração observada nos exames bioquímicos realizados com concentrações de cálcio total $(9,7 \mathrm{mg} / \mathrm{dL}-8$ a 12 $\mathrm{mg} / \mathrm{dL})$ e iônico $(5,64 \mathrm{mg} / \mathrm{dL}-4,3$ A 5,9 mg/dL) dentro dos parâmetros normais para a espécie. As radiografias revelaram a diminuição generalizada da opacidade óssea com adelgaçamento das corticais dos ossos longos, sugerindo um quadro de osteopenia. Observou-se ainda, fratura no terço proximal da diáfise femoral direita, fratura no terço médio da diáfise femoral esquerda (Figuras 1 e 2 ) e fratura compressiva da última vertebra torácica (Figura 2).

Este caso foi diagnosticado como hiperparatiroidismo secundário nutricional, com base em critérios clínicos, radiográficos, bioquímicos e histórico da dieta do animal. $\mathrm{O}$ animal foi submetido à enema e massagem abdominal, durante os dois primeiros dias, a fim de eliminar o excesso de fezes retido. Foi tratado em confinamento em uma caixa, a alimentação caseira foi substituída por ração comercial amolecida até ficar pastosa, óleo mineral $1 \mathrm{ml}$ BID até voltar a defecar regularmente, suplemento a base de cálcio e vitamina D (cal D mix ${ }^{\circledR} 1 \mathrm{ml}$, SID, por 30 dias), cloridrato de tramadol $2,5 \mathrm{mg} / \mathrm{kg}$ TID durante 15 dias e cetoprofeno $\mathrm{mg} / \mathrm{kg}$ SID durante 3 dias. O proprietário foi ainda instruído a realizar massagem abdominal 3 vezes ao dia, afim de auxiliar o animal a defecar e urinar.

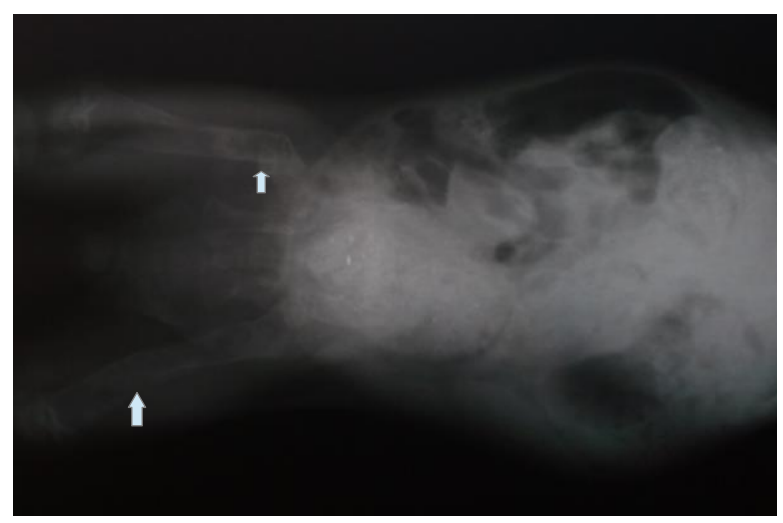

Figura 1. Projeção radiográfica ventrodorsal de felino. Observa-se diminuição generalizada da opacidade óssea com adelgaçamento das corticais dos ossos longos, fratura em terço proximal da diáfise femoral direita, (seta menor) e fratura em galho verde no terço médio da diáfise femoral esquerda (seta maior).

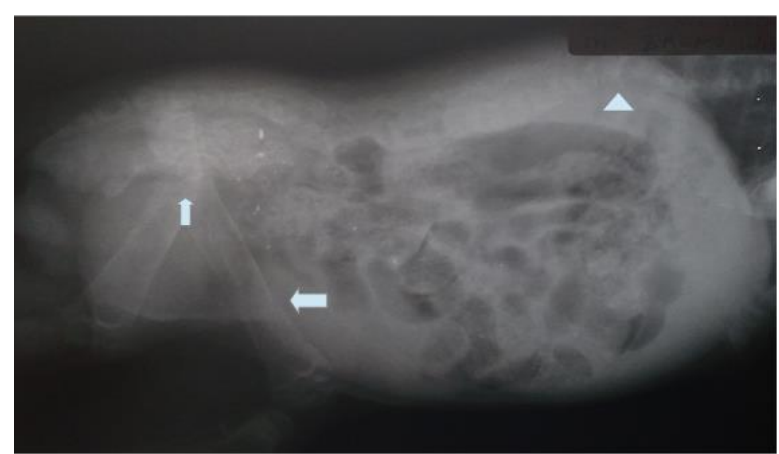

Figura 2. Projeção radiográfica laterolateral de felino. Observa-se diminuição generalizada da opacidade óssea com adelgaçamento das corticais dos ossos longos, fratura em terço proximal da diáfise femoral direita, (seta menor), fratura em galho verde no terço médio da diáfise femoral esquerda (seta maior) e fratura compressiva da última vertebra torácica (ponta de seta).

Após 30 dias do início do tratamento, o animal apresentou redução significativa do déficit neurológico, defecando e urinando regularmente e apresentando respostas adequadas aos testes de propriocepção, dor superficial e profunda. O animal já conseguia se apoiar nos membros pélvicos e caminhar pequenas distâncias, uma nova avaliação radiográfica revelou a formação de calos ósseos e melhora na mineralização óssea (Figura 3 e 4), o proprietário foi instruído a estimular o animal movimentando os membros e ajudando ele a permanecer em estação. Quatro 
meses após o início do tratamento, o animal foi reavaliado o mesmo já caminhava ativamente. Foi realizado novo exame radiográfico afim de avaliar a resolução das fraturas e a mineralização óssea, observando-se completa cicatrização dos ossos longos, com mineralização adequada da estrutura óssea, persistência de deformidade nos fêmures, desvio postural na coluna torácica e deformidade na décima segunda e décima terceira vertebras torácicas (Figura 5), no entanto, o animal apresentava-se clinicamente saudável não sendo observada claudicação ou qualquer outra alteração clínica e recebeu alta médica.

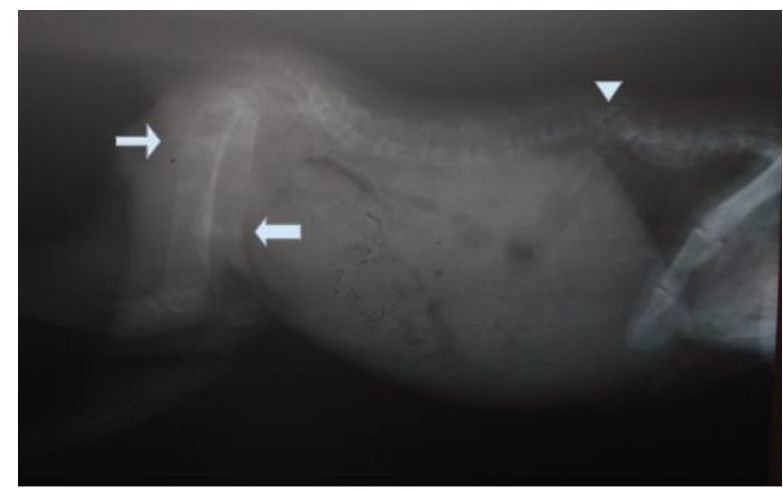

Figura 3. Projeção radiográfica laterolateral de felino após 30 dias de tratamento. Observa-se calo ósseo em fratura no terço proximal da diáfise femoral direita (seta menor), no terço médio da diáfise femoral esquerda (seta maior) e deformidade da última vertebra torácica (ponta de seta).

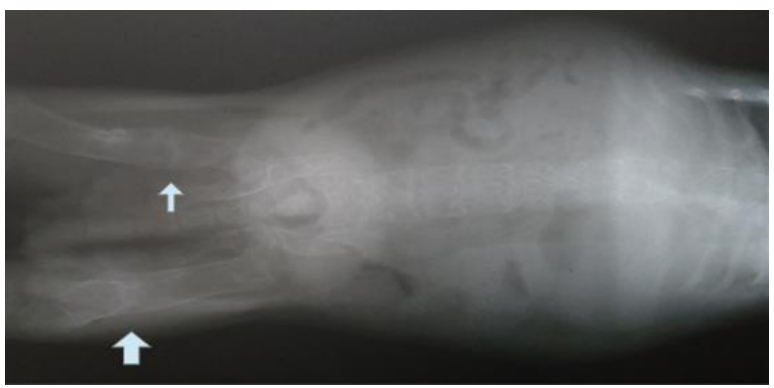

Figura 4. Projeção radiográfica ventrodorsal de felino após 30 dias de tratamento. Observa-se formação de calo ósseo em fratura no terço proximal da diáfise femoral direita (seta menor) e no terço médio da diáfise femoral esquerda (seta maior).

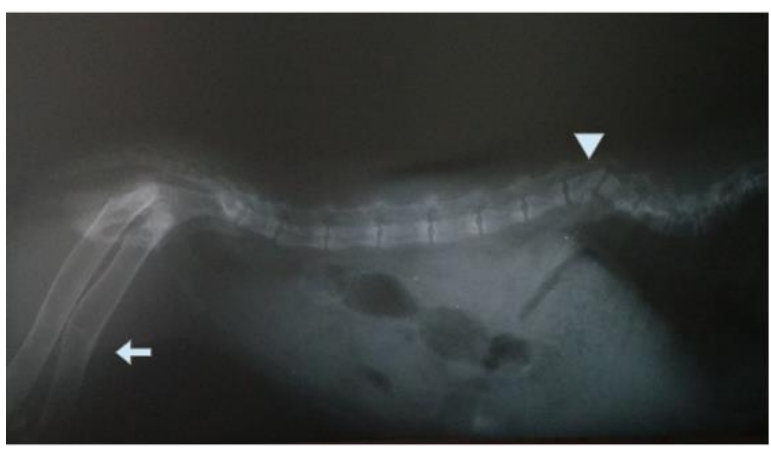

Figura 5. Projeção radiográfica laterolateral de felino após 4 meses do primeiro atendimento. Observa-se deformidades ósseas em fêmur (seta) e na última vértebra torácica (ponta de seta).

\section{Discussão}

O uso de comida caseira em filhotes em fase de crescimento descrita no presente estudo já constituiu uma causa comum para HSN em felinos, no entanto tem se tornado cada vez mais raro nos dias atuais em função da difusão de alimentos industriais balanceados (Tomsa et al., 1999; Dimopoulou et al., 2010). Apesar da difusão destes alimentos a um baixo custo, observa-se uma ocorrência esporádica de casos de HSN em felinos domésticos. Este fato tem sido atribuído por alguns autores à falsa crença de que dietas a base de carne correspondem às presas naturais destes animais, constituindo assim uma alimentação adequada (Martin \& Capen, 1985; Dimopoulou et al., 2010). Martin \& Capen (1985) relataram que a proporção de Ca e $\mathrm{P}$ no coração e fígado bovinos é de 1:20 e 1:50, respectivamente, demonstrando o quão falsa e prejudicial pode ser essa premissa. Há relatos de diversos autores de que gatos jovens submetidos à alimentação a base de carne desenvolveram HSN (Krook et al., 1963; Sturion \& Pereira, 1995; Tomsa et al., 1999; Rahal et al., 2002).

O diagnóstico do HNS baseia-se na presença de sinais clínicos, achados bioquímicos como alterações na concentração séricas de cálcio e fósforo, a atividade da enzima fosfatase alcalina aumentada, dosagem sérica de paratormônio, exames de imagem avaliando osteopenia e presença de fraturas patológicas (Krook et al., 1963; Tomsa et al., 1999; Moarrabi et al., 2008; Dimopoulou et al., 2010).

No presente trabalho, observou-se concentrações fisiológicas de $\mathrm{Ca}$ e discreto aumento da concentração sérica de $\mathrm{P}$ embora esse aumento da concentração de fósforo possa ser interpretado como um desequilíbrio da relação de $\mathrm{Ca}$ e $\mathrm{P}$ é descrito na literatura concentrações elevadas de $\mathrm{P}$ como um achado comum em filhotes saudáveis (Hazewinkel, 1994), tornando este achado pouco relevante e corroborando com a literatura que afirma que as concentrações plasmáticas desses elementos contribuem pouco para o diagnóstico desta patologia em felinos (Rahal et al., 2002; Moarrabi et al., 2008). A dosagem da fosfatase alcalina constitui um bom indicador de atividade osteoclástica (Krook et al., 1963). No entanto, ainda é um achado pouco especifico visto que esta enzima também se relaciona com lesões em outros sistemas. A análise das concentrações do hormônio da paratireoide é provavelmente a dosagem de maior valor 
diagnóstico para hiperparatireoidismo, embora não apresente informações sobre a origem deste. Apesar do valor diagnóstico deste exame ele é pouco rotineiro devido ao custo elevado e por requerer laboratórios especializados (Hazewinkel, 1994; Tomsa et al., 1999; Rahal et al., 2002) não tendo sido dosado no presente caso por limitação financeira do proprietário.

O presente estudo obteve alterações radiográficas compatíveis com um quadro de osteopenia generalizado. A realização de exame radiográfico afim de identificar fraturas patológicas e osteopenia é o exame mais explorado para o diagnóstico de HSN em felinos (Sturion \& Pereira, 1995; Tomsa et al., 1999; Moarrabi et al., 2008; Meireles et al., 2012). Embora as radiografias convencionais sejam aceitas pela maioria dos autores como adequadas para o diagnóstico de osteopenia nos quadros de HSN, Dimopoulou et al. (2010) defendem que as radiografias convencionais têm baixa sensibilidade na detecção da perda óssea, exigindo perda óssea elevada para possibilitar o diagnóstico de osteopenia, além de constituir uma avaliação subjetiva não quantificável, os autores sugerem, portanto, o uso de técnicas mais sofisticadas para tal como a densitometria por DEXA (dual energy x-ray absorptiometry) e a tomografia computadorizada (CT). Rahal et al. (2002) reconheceram a limitação das radiografias convencionais para o diagnóstico de osteopenia e apresenta uma alternativa mais barata para realização de densitometria óssea em felinos por meio do método de densitometria óptica em imagens radiográficas. No entanto a realização deste exame em felinos ainda está em fase de padronização não sendo muito difundido.

$\mathrm{O}$ uso de alimento comercial de boa qualidade tem sido descrito na literatura como tratamento adequado do HSN não havendo necessidade de suplementação com Ca (Dimopoulou et al., 2010). No entanto, Wing (1967) demonstrou experimentalmente uma maior eficiência do tratamento do HSN em felinos a partir da ingestão de dietas com proporção de cálcio para fósforo elevada, $3: 1$ ou $2: 1$, propiciando um período de recuperação mais breve que os animais alimentados com dieta de proporção 1:1, geralmente utilizada nas formulações das rações comerciais adequadas ao crescimento normal de felinos saudáveis (Kirk et al., 2000). Sendo, portanto, favorável ao tratamento à suplementação de cálcio além da correção da alimentação (Watson, 1983) optou-se por fazer a suplementação de cálcio visando reduzir o tempo de recuperação do animal.

A abordagem terapêutica adotada de restrição de movimentos pelo confinamento constitui uma abordagem eficaz em casos de HSN com resolução espontânea das fraturas (Tomsa et al., 1999; Dimopoulou et al., 2010) constando na literatura apenas um relato onde fixação externa foi associada a restrição de espaço (Nagata \& Yuki, 2013) não tendo sido necessária no presente caso.

Não constam relatos na literatura de animais com lesões de coluna similares às descritas no presente trabalho que tenham sido reabilitados, sendo considerado o déficit neurológico secundário a lesão de coluna irreversível, no entanto, o presente trabalho demonstrou que mediante mineralização adequada e tratamento de suporte que permita ao animal manter suas funções fisiológicas durante o tratamento, pode-se obter a estabilização da coluna com cicatrização das vértebras lesionadas.

\section{Conclusão}

O HSN em felinos jovens, embora venha se tornando incomum, ainda representa uma enfermidade importante na clínica de felinos como evidenciado no presente trabalho. A realização de exame radiográfico de rotina associado ao histórico do animal forneceu dados suficientes para obtenção do diagnóstico clínico de HSN. O tratamento através de correção alimentar com suplementação de cálcio mostrou-se adequado para a reversão do quadro ósseo-metabólico. $\mathrm{O}$ confinamento associado à analgesia, uso de laxante e massagens abdominais mostrou-se eficiente para manutenção das funções fisiológicas normais e permitindo a completa recuperação do animal.

\section{Referências Bibliográficas}

Dimopoulou, M., Kirpensteijn, J., Nielsen, D.H., Buelund, L., Hansen, M.S., 2010. Nutritional secondary hyperparathyroidism in two cats. Veterinary and Comparative Orthopaedics and Traumatology 23, 56-61.

Hazewinkel, H.A.W., 1994. Skeletal disease, In: Wills, J.M., Simpson, K.W. (Eds.), The Waltham book of clinical nutrition of the dog and cat, Pergamon, Grã Bretanha.

Jubb, K.V.F., P.C., K., N., P., M.G., M., 2007. Pathology of domestic animals. Academic press, San Diego. 
Kirk, C.A., Debwekeler, J., Armstrong, P.J., 2000. Normal cats, In: S., H.M., D., T.C., Remillard, R.L., Roundebush, P. (Eds.), Small animal clinical nutrition, Walsworth Publishing Company, Missouri, USA.

Krook, L., Barrett, R.B., Usui, K., Wolke, R.E., 1963. Nutritional secondary hyperparathyroidism in the cat. Cornell Veterinarian 53, 224-240.

Martin, S.L., Capen, C.C., 1985. The endocrine system, In: Pratt, P.W. (Ed.), Feline medicine, American Veterinary, Santa Barbara. USA.

Meireles, Y.S., Rondon, É.L., Souza, A.C.S., Menegassi, C.C., Correia, C.Y., Néspoli, P.B., 2012. Aspectos radiográficos do hiperparatireoidismo nutricional em uma suçuarana (Puma concolor). Acta Scientiae Veterinariae 40, 1-4.

Moarrabi, A., Mosallanejad, B., Khadjeh, G., Noorani, B., 2008. Nutritional secondary hyperparathyroidism in cats under six-monthold of Ahvaz. Iranian Journal of Veterinary Surgery 3, 59-65.

Nagata, N., Yuki, M., 2013. Nutritional secondary hyperparathyroidism in a cat. Journal of Animal Clinical Medicine 22, 101-104.

Prestes, R.S., Oliveira, P.D., Silva, M.G.N., Pilotto, B.M., Mistieri, M.L.A., Pascon, E.J.P., Machado, I.R.L., 2016. Hiperparatireoidismo secundário nutricional em felino. Caso, $37^{\circ}$ Congresso Brasileiro da Anclivepa, Goiânia, Goiás.

Queiroz, D.J., Bernardi, N., Dias, D.P.M., Cadioli, F.A., 2015. Hiperparatireoidismo nutricional secundário em equinos e ruminantes: Revisão de literatura. Nucleus Animalium 7, 51-57.

Rahal, S.C., Mortari, A.C., Caporali, E.H.G., Vulcano, L.C., Marques dos Santos, F.A., Takahira, R.K., Crocci, A.J., 2002. Densitometria óptica radiográfica na avaliação do hiperparatireoidismo secundário nutricional induzido em gatos jovens. Ciência Rural 32, 421-425.

Sturion, D.J., Pereira, P.M., 1995. Diagnóstico radiográfico de caninos e felinos com hiperparatireoidismo nutricional secundário. Semina: Ciências Agrárias 16, 28-33.

Tomsa, K., Glaus, T., Hauser, B., Flückiger, M., Arnold, P., Wess, G., Reusch, C., 1999. Nutritional secondary hyperparathyroidism in six cats. Journal of Small Animal Practice 40, 533-539.

Watson, A.D.J., 1983. Treatment of nutritional secondary hyperparathyroidism in the cat. The Canadian Veterinary Journal 24, 107.

Wing, P.C., 1967. The reversibility of nutritional osteitis fibrosa. Dissertation Abstracts: B 27, 3735-3736.3735B-3736B.

Recebido: 10 Abr. 2018

Aprovado: 1 Mai. 2018

Publicado: 14 Jul. 2018

Licenciamento: Este artigo é publicado na modalidade Acesso Aberto sob a licença Creative Commons Atribuição 4.0 (CC-BY 4.0), a qual permite uso irrestrito, distribuição, reprodução em qualquer meio, desde que o autor e a fonte sejam devidamente creditados. 